\title{
On a sign-changing solution for some fractional differential equations
}

\section{Kemei Zhang* ${ }^{*}$}

\section{"Correspondence:} zhkm90@126.com

School of Mathematics Sciences, Qufu Normal University, Qufu,

Shandong 273165, China

\section{Springer}

\begin{abstract}
In this paper, a kind of $\alpha$ th $(3<\alpha \leq 4)$ order differential equation with two-point boundary conditions is considered. The existence result of a sign-changing solution is given by the topological degree theory and the fixed point index theory.
\end{abstract}

MSC: 34B15

Keywords: sign-changing solution; topological degree; fixed point index

\section{Introduction}

Fractional differential equations have gained much importance and attention due to the fact that they have been proved to be valuable tools in the modeling of many phenomena in engineering and sciences such as physics, mechanics, economics and biology; see [1-3]. In recent years, there has been a great deal of research on the existence and/or uniqueness of solution to boundary value problems for fractional-order differential equations. By means of Leray-Schauder degree theory, fixed point theorem on cone, a monotone iterative method combined with lower and upper solutions or by introducing height functions of the nonlinear term on some bounded sets and considering integrations of these height functions, several local existence and multiplicity results of solutions are obtained, for details, please refer to [4-11] and the references therein. In [8-11], the authors studied the following $\alpha$ th $(3<\alpha \leq 4)$ order differential equation:

$$
D_{0^{+}}^{\alpha} u(t)=f(t, u(t)), \quad t \in(0,1),
$$

with different boundary conditions. By using the Leray-Schauder nonlinear alternative theorem, fixed point index theory, the properties of cone and fixed point theorems for a mixed monotone operator, the existence results of positive solutions for singular and nonsingular nonlinear fractional differential equation boundary value problems are obtained.

To the best of our knowledge, there are less papers studying the existence of signchanging solution for the fractional differential equations. The aim of this paper is to investigate the existence of sign-changing solution for the following Riemann-Liouville

(c) The Author(s) 2017. This article is distributed under the terms of the Creative Commons Attribution 4.0 International License (http://creativecommons.org/licenses/by/4.0/), which permits unrestricted use, distribution, and reproduction in any medium, provided you give appropriate credit to the original author(s) and the source, provide a link to the Creative Commons license, and indicate if changes were made. 
fractional differential equations with two-point boundary conditions:

$$
\left\{\begin{array}{l}
D_{0^{+}}^{\alpha} u(t)=f(t, u(t)), \quad t \in(0,1), \\
u(0)=u(1)=u^{\prime}(0)=u^{\prime}(1)=0,
\end{array}\right.
$$

where $D_{0^{+}}^{\alpha}$ is the standard Riemann-Liouville fractional derivative of order $3<\alpha \leq 4$ defined by

$$
D_{0^{+}}^{\alpha} u(t)=\frac{1}{\Gamma(4-\alpha)}\left(\frac{d}{d t}\right)^{4} \int_{0}^{t} \frac{u(s)}{(t-s)^{\alpha-3}} d s
$$

$\Gamma$ denotes the Euler gamma function, provided that the right side is point wise defined on $(0,+\infty)$. The existence result of a sign-changing solution is given by topological degree theory and fixed point index theory. One can refer for the method and the theoretical knowledge used in this paper to [12-18].

\section{Preliminaries and some lemmas}

Definition 2.1 $([13,14])$ Let $E$ be a real Banach space and $A: E \rightarrow E$ be a nonlinear operator. A nonzero solution to the equation $x=\lambda A x$ is called an eigenvector of the nonlinear operator $A$; the corresponding number $\lambda$ is called a characteristic value of $A$, and $\lambda^{-1}$ is called a eigenvalue of $A$.

Definition 2.2 $([14,16])$ Let $E_{1}, E_{2}$ be real Banach spaces and $D \subset E_{1}$ contain the outside of a ball $\{x \mid\|x\| \leq r\}, A: D \rightarrow E_{2}$. The operator $A$ is called asymptotically linear, if there is a continuous linear operator $B: E_{1} \rightarrow E_{2}$ such that

$$
\lim _{\|x\| \rightarrow \infty} \frac{\|A x-B x\|}{\|x\|}=0 .
$$

The operator $B$ involved in the definition of an asymptotically linear operator $A$ is uniquely determined, it is called the derivative of $A$ at infinity and is denoted by $A^{\prime}(\infty)$.

Lemma 2.1 ([16]) Let E be a Banach space and $P$ be a total cone in E. Suppose $T: P \rightarrow P$ is a bounded linear operator (therefore, $T$ can be uniquely extended to a bounded linear operator on $\overline{P-P}=E$, and the extended operator is denoted by $T$ again) with the spectral radius $r(T)<1$. If $w, w_{0} \in E$ such that $w \leq T w+w_{0}$, then

$$
w \leq(I-T)^{-1} w_{0},
$$

where $(I-T)^{-1}$ is the inverse operator of the operator $I-T$.

Lemma $2.2([13,16])$ Suppose that $E$ is a Banach space, $A: E \rightarrow E$ is a completely continuous and asymptotically linear operator. If 1 is not the eigenvalue of the linear operator $A^{\prime}(\infty)$, then there exists $R_{0}>0$ such that

$$
\operatorname{deg}\left(I-A, B_{R}, \theta\right)=(-1)^{\gamma}
$$

for any $R \geq R_{0}$, where $B_{R}=\{x \in E \mid\|x\|<R\}, \gamma$ is the sum of the algebraic multiplicities of the real eigenvalues of $A^{\prime}(\infty)$ in $(1,+\infty)$. 
Lemma 2.3 ([16]) Let $E$ be a Banach space and $\Omega$ be a bounded open set in $E$ with $\theta \in \Omega$. Suppose that $A: \bar{\Omega} \rightarrow E$ is a completely continuous operator. If

$$
A u \neq \mu u, \quad \forall u \in \partial \Omega, \mu \geq 1,
$$

then the topological degree $\operatorname{deg}(I-A, \Omega, \theta)=1$.

Lemma 2.4 ([14]) Let $E$ be a Banach space, and $P$ be a cone in $E$, and $\Omega$ be a bounded open set in $E$. Suppose that $A: P \cap \bar{\Omega} \rightarrow P$ is a completely continuous operator. If

$$
A u \neq \mu u, \quad \forall u \in P \cap \partial \Omega, \mu \geq 1
$$

then the fixed point index $i(A, P \cap \Omega, P)=1$.

Remark 2.1 Let $E$ be a Banach space and $\Omega$ be a bounded open set in $E$ with $\theta \in \Omega$. Suppose that $A: \bar{\Omega} \rightarrow E$ is a completely continuous operator. If

$$
\|A u\|<\|u\|, \quad \forall u \in \partial \Omega
$$

then the topological degree $\operatorname{deg}(I-A, \Omega, \theta)=1$. Furthermore, suppose that $P$ is a cone in $E$ and satisfies

$$
\|A u\|<\|u\|, \quad \forall u \in \pm P \cap \partial \Omega
$$

then the fixed point index $i(A, \pm P \cap \Omega, \pm P)=1$.

Given $h \in C[0,1]$, it follows from [8] that the unique solution of the problem

$$
\left\{\begin{array}{l}
D_{0^{+}}^{\alpha} u(t)+h(t)=0, \quad 0<t<1, \\
u(0)=u(1)=u^{\prime}(0)=u^{\prime}(1)=0,
\end{array}\right.
$$

can be expressed uniquely by $u(t)=\int_{0}^{1} G(t, s) h(s) d s$, where

$$
G(t, s)=\frac{1}{\Gamma(\alpha)}\left\{\begin{array}{c}
(t-s)^{\alpha-1}+t^{\alpha-2}(1-s)^{\alpha-2}[(s-t)+(\alpha-2)(1-t) s] \\
0 \leq s \leq t \leq 1 \\
t^{\alpha-2}(1-s)^{\alpha-2}[(s-t)+(\alpha-2)(1-t) s] \\
0 \leq t \leq s \leq 1
\end{array}\right.
$$

It is easy to verify that $G(t, s)>0$ for $t, s \in(0,1)$ and

$$
(\alpha-2) t^{\alpha-2}(1-t)^{2} s^{2}(1-s)^{\alpha-2} \leq \Gamma(\alpha) G(t, s) \leq M_{0} t^{\alpha-2}(1-t)^{2}, \quad \forall t, s \in[0,1],
$$

where $M_{0}=\max \left\{\alpha-1,(\alpha-2)^{2}\right\}$.

In the following, we introduce some notations. Let $X=C[0,1]$ and

$$
P=\{x \in X \mid x(t) \geq 0, t \in[0,1]\},
$$


then $P$ is a cone in $X$. Set $e(t)=t^{\alpha-2}(1-t)^{2}$,

$$
\begin{aligned}
& X_{e}=\{x \in X \mid \text { there exists } \lambda>0 \text { such that }-\lambda e \leq x \leq \lambda e\}, \\
& \|x\|_{e}=\inf \{\lambda>0 \mid-\lambda e \leq x \leq \lambda e\}, \quad \forall x \in E_{e}
\end{aligned}
$$

then $\|x\|_{e}$ is called the e-norm of the element $x \in X_{e}$. It is easy to see that $X_{e}$ becomes a Banach space under the norm $\|\cdot\|_{e}$ and $P_{e}=P \cap X_{e}$ is a normal solid cone in $X_{e}\left(e \in \stackrel{\circ}{P}_{e}\right)$.

Before proving the following lemmas, we need the following conditions:

$\left(\mathrm{H}_{1}\right) f:[0,1] \times(-\infty,+\infty) \rightarrow(-\infty,+\infty)$ is continuous and $f(t, x) x>0$ for all $x \in R \backslash\{0\}$ and $t \in[0,1]$.

$\left(\mathrm{H}_{2}\right) \lim _{x \rightarrow \infty} \frac{f(t, x)}{x}=\beta_{\infty}(t)$ uniformly with respect to $t \in[0,1]$.

Evidently, $\beta_{\infty}(t) \in C[0,1]$ and $\beta_{\infty}(t) \geq 0$ for $t \in[0,1]$. Define the operators $K, F$ such that

$$
\begin{aligned}
& K u(t)=\int_{0}^{1} G(t, s) u(s) d s, \quad u \in X, t \in[0,1], \\
& (F u)(t)=f(t, u(t)), \quad t \in[0,1], u \in X,
\end{aligned}
$$

and $A=K F$.

Lemma 2.5 The operator $K$ defined by (2.3) satisfies $K: X \rightarrow X_{e}$ and $K: P \backslash\{\theta\} \rightarrow \stackrel{\circ}{P}_{e}$, where $\stackrel{\circ}{P}_{e}=\{x \in X \mid$ there exist $\tilde{\alpha}>0, \tilde{\beta}>0$ such that $\tilde{\alpha} e \leq x \leq \tilde{\beta} e\}$.

Proof For any $u \in X$, by (2.2), (2.3) we have

$$
\begin{aligned}
& K u(t)=\int_{0}^{1} G(t, s) u(s) d s \leq \frac{M_{0}}{\Gamma(\alpha)} \int_{0}^{1}|u(s)| d s \cdot e(t)=\tilde{\lambda} e(t), \quad t \in[0,1], \\
& K u(t)=\int_{0}^{1} G(t, s) u(s) d s \geq \int_{0}^{1} G(t, s)(-|u(s)|) d s \geq-\tilde{\lambda} e(t), \quad t \in[0,1],
\end{aligned}
$$

where $\tilde{\lambda}=\frac{M_{0}}{\Gamma(\alpha)} \int_{0}^{1}|u(s)| d s$, i.e., $K: X \rightarrow X_{e}$.

Moreover, for $u \in P \backslash\{\theta\}$, by (2.2), one can get

$$
K u(t) \geq \frac{\alpha-2}{\Gamma(\alpha)} \int_{0}^{1} s^{2}(1-s)^{\alpha-2} u(s) d s \cdot e(t), \quad t \in[0,1],
$$

then it follows from (2.5), (2.7) that $\tilde{\alpha} e(t) \leq K u(t) \leq \tilde{\beta} e(t)$, where

$$
\tilde{\alpha}=\frac{\alpha-2}{\Gamma(\alpha)} \int_{0}^{1} s^{2}(1-s)^{\alpha-2} u(s) d s, \quad \tilde{\beta}=\frac{M_{0}}{\Gamma(\alpha)} \int_{0}^{1} u(s) d s,
$$

i.e., $K u \in \stackrel{\circ}{P}_{e}$, and so we show that $K: P_{e} \backslash\{\theta\} \rightarrow \stackrel{\circ}{P}_{e}$.

Lemma 2.6 Suppose that $\left(\mathrm{H}_{2}\right)$ holds, then the operator $A: X_{e} \rightarrow X_{e}$ is asymptotically linear, and the derivative of $A$ at infinity $A^{\prime}(\infty)=B$, where

$$
B u(t)=\int_{0}^{1} G(t, s) \beta_{\infty}(s) u(s) d s, \quad u \in X_{e} .
$$


Proof It follows from Lemma 2.5 and the definitions of $A, B$ that $A, B: X_{e} \rightarrow X_{e}$. By $\left(\mathrm{H}_{2}\right)$ we know that, for any $\varepsilon>0$, there exists $l>0$ such that

$$
\left|\frac{f(t, x)}{x}-\beta_{\infty}(t)\right|<\varepsilon
$$

for any $x$ with $|x| \geq l$ and $t \in[0,1]$.

Set $T_{l}=\left\{x \in X_{e} \mid\|x\|_{e} \leq l\right\}$, and let

$$
M=\sup _{x \in T_{l}}\left\{\|A x\|_{e},\|B x\|_{e}\right\}
$$

For $u \in X_{e}$, define

$$
\begin{aligned}
& \phi_{1}(u(t))= \begin{cases}u(t), & |u(t)| \leq l, \\
l, & |u(t)| \geq l,\end{cases} \\
& \phi_{2}(u(t))= \begin{cases}l, & |u(t)| \leq l, \\
u(t), & |u(t)| \geq l\end{cases}
\end{aligned}
$$

Then

$$
\begin{aligned}
& A u(t)=A \phi_{1}(u(t))+A \phi_{2}(u(t))-A l(t), \quad t \in[0,1], u \in X_{e} \\
& B u(t)=B \phi_{1}(u(t))+B \phi_{2}(u(t))-B l(t), \quad t \in[0,1], u \in X_{e}
\end{aligned}
$$

where $l(t) \equiv l$. It follows from (2.9) that

$$
\left\|A \phi_{1}(u)\right\|_{e} \leq M, \quad\|A l\|_{e} \leq M, \quad\left\|B \phi_{1}(u)\right\|_{e} \leq M, \quad\|B l\|_{e} \leq M
$$

Therefore, by (2.10), (2.11) and (2.12), we have

$$
\|A u-B u\|_{e} \leq 4 M+\left\|\left(A \phi_{2}\right) u-\left(B \phi_{2}\right) u\right\|_{e}
$$

From (2.8), we get

$$
\begin{aligned}
& \left|\left(A \phi_{2}\right) u(t)-\left(B \phi_{2}\right) u(t)\right| \\
& \quad=\left|\int_{0}^{1} G(t, s)\left[f\left(s, \phi_{2}(u(s))\right)-\beta_{\infty}(s) \phi_{2}(u(s))\right] d s\right| \\
& \quad \leq \int_{0}^{1} G(t, s)\left|f\left(s, \phi_{2}(u(s))\right)-\beta_{\infty}(s) \phi_{2}(u(s))\right| d s \\
& \quad \leq \frac{M_{0}}{\Gamma(\alpha)} \varepsilon\|u\|_{e} e(t)
\end{aligned}
$$

for any $u \in X_{e}$, from which one deduces that

$$
\left\|\left(A \phi_{2}\right) u-\left(B \phi_{2}\right) u\right\| \leq \frac{M_{0}}{\Gamma(\alpha)} \varepsilon\|u\|_{e} e(t),
$$


and therefore from (2.13), one can get

$$
\lim _{\|u\|_{e} \rightarrow \infty} \frac{\|A u-B u\|_{e}}{\|u\|_{e}}=0
$$

i.e., the operator $A$ is asymptotically linear, and $A^{\prime}(\infty)=B$.

In this paper, we always denote by $\Omega_{r}=\left\{u \in X:\|u\|_{e}<r\right\}(r>0)$ the open ball of radius $r$ and by $\theta$ the zero function in $X_{e}$. For the concepts and properties on the cone and the topological degree, one can refer to $[12,14,16]$.

\section{Main results}

Theorem 3.1 Suppose that we have the conditions $\left(\mathrm{H}_{1}\right),\left(\mathrm{H}_{2}\right)$ and the following conditions:

$\left(\mathrm{H}_{3}\right)$ There is $p>0$ such that $|u| \leq p, t \in[0,1]$ imply that $|f(t, u)|<\eta p$, where $\eta=\frac{\Gamma(\alpha)}{M_{0}}$.

$\left(\mathrm{H}_{4}\right) \sup _{t \in[0,1]} \beta_{\infty}(t)<\lambda_{1}$, where $\lambda_{1}$ is the first characteristic value of $K$ defined by (2.3).

If 1 is not the characteristic value of $B$ and the sum of the algebraic multiplicities $\gamma$ of the real eigenvalues of $B$ in $(1,+\infty)$ is odd, then the BVP (1.1) has at least a sign-changing solution.

Proof Evidently, $A: X_{e} \rightarrow X_{e}$ is completely continuous. By $\left(\mathrm{H}_{1}\right)$ and Lemma 2.5, we know that $A: P_{e} \backslash\{\theta\} \rightarrow \stackrel{\circ}{P}_{e}$. From $\left(\mathrm{H}_{3}\right)$, for any $u \in \partial \Omega_{p}$, we have

$$
\begin{aligned}
& |A u(t)| \\
& =\left|\int_{0}^{1} G(t, s) f(s, u(s)) d s\right| \\
& \quad \leq \int_{0}^{1} G(t, s)|f(s, u(s))| d s \\
& \quad<\frac{M_{0} \eta}{\Gamma(\alpha)} t^{\alpha-2}(1-t)^{2} \cdot\|u\|_{e} \\
& =\frac{M_{0} \eta}{\Gamma(\alpha)} \cdot\|u\|_{e} \cdot e(t) \\
& =\|u\|_{e} \cdot e(t),
\end{aligned}
$$

from which one deduces that $\|A u\|_{e}<\|u\|_{e}$, so by Remark 2.1, we get

$$
\begin{aligned}
& \operatorname{deg}\left(I-A, \Omega_{p}, \theta\right)=1, \\
& i\left(A, \pm P_{e} \cap \Omega_{p}, \pm P_{e}\right)=1 .
\end{aligned}
$$

From conditions $\left(\mathrm{H}_{2}\right)$ and $\left(\mathrm{H}_{4}\right)$, there exist $\varepsilon_{1}>0$ small enough and $R_{1}>p$ large enough such that

$$
f(t, u)<\left(\lambda_{1}-\varepsilon_{1}\right) u, \quad \text { for any } u \geq R_{1} \text { and } t \in[0,1] \text {. }
$$

Let

$$
C_{1}=\max _{0 \leq u \leq R_{1}, 0 \leq t \leq 1}\left|f(t, u)-\left(\lambda_{1}-\varepsilon_{1}\right) u\right|+1,
$$


then it follows from (3.4) that

$$
|f(t, u)| \leq\left(\lambda_{1}-\varepsilon_{1}\right)|u|+C_{1}, \quad \forall u \in[0,+\infty) \text { and } t \in[0,1]
$$

Let $D=\left\{u \in P_{e}: A u=\lambda u, \lambda \geq 1\right\}$. In the following, we shall prove that $D$ is bounded. In fact, if $u \in D$, then by (3.5), there exists $\lambda \geq 1$ such that

$$
\begin{aligned}
0 & \leq u(t) \leq \lambda u(t)=A u(t) \\
& =\int_{0}^{1} G(t, s) f(s, u(s)) d s \\
& \leq\left(\lambda_{1}-\varepsilon_{1}\right) \int_{0}^{1} G(t, s) u(s) d s+C_{1} \int_{0}^{1} G(t, s) d s \\
& =\left(\lambda_{1}-\varepsilon_{1}\right)(K u)(t)+\phi(t),
\end{aligned}
$$

where $\phi(t)=C_{1} \int_{0}^{1} G(t, s) d s \in P_{e}$. Since $r\left(\left(\lambda_{1}-\varepsilon_{1}\right) K\right)<1$, from Lemma 2.1 we have $u(t) \leq$ $\left(I-\left(\lambda_{1}-\varepsilon_{1}\right) K\right)^{-1} \phi(t)$, and then by (2.2), we get

$$
-\frac{C_{1} M_{0}}{\Gamma(\alpha)} \cdot e(t) \leq-\phi(t) \leq\left(I-\left(\lambda_{1}-\varepsilon_{1}\right) K\right) u(t) \leq \phi(t) \leq \frac{C_{1} M_{0}}{\Gamma(\alpha)} \cdot e(t),
$$

which, together with the definition of $\|\cdot\|_{e}$, implies that

$$
\left\|\left(I-\left(\lambda_{1}-\varepsilon_{1}\right) K\right) u\right\|_{e} \leq \frac{C_{1} M_{0}}{\Gamma(\alpha)}
$$

and thus

$$
\begin{aligned}
\|u\|_{e} & =\left\|\left(I-\left(\lambda_{1}-\varepsilon_{1}\right) B\right)^{-1}\left[\left(I-\left(\lambda_{1}-\varepsilon_{1}\right) B\right) u\right]\right\|_{e} \\
& \leq\left\|\left(I-\left(\lambda_{1}-\varepsilon_{1}\right) B\right)^{-1}\right\| \cdot \frac{C_{1} M_{0}}{\Gamma(\alpha)} .
\end{aligned}
$$

So $D$ is bounded and then there exists a sufficiently large number $R_{2}>p$ such that, for any $R \geq R_{2}$, one can get

$$
A u \neq \lambda u, \quad \forall u \in P_{e} \cap \partial \Omega_{R} \text { and } \lambda \geq 1,
$$

which together with Lemma 2.4 implies that

$$
i\left(A, P_{e} \cap \Omega_{R}, P_{e}\right)=1, \quad R \geq R_{2} .
$$

Similar to the proof of (3.9), there exists $R_{3}>p$ such that, for $R \geq R_{3}$, we have

$$
i\left(A,\left(-P_{e}\right) \cap \Omega_{R},-P_{e}\right)=1 .
$$

For any $R_{4}>\left\{R_{2}, R_{3}\right\}$, it follows from (3.3), (3.9) and (3.10) and the excision property of fixed point index that

$$
i\left(A,\left( \pm P_{e}\right) \cap\left(\Omega_{R_{4}} \backslash \overline{\Omega_{p}}\right), \pm P_{e}\right)=1-1=0 .
$$


Since $A$ has no fixed point on $\partial\left( \pm P_{e} \backslash\{\theta\}\right),(3.11)$ and the permanence of the topological degree imply that

$$
\operatorname{deg}\left(I-A, \pm \stackrel{\circ}{P}_{e} \cap\left(\Omega_{R_{4}} \backslash \overline{\Omega_{p}}\right), \theta\right)=i\left(A,\left( \pm P_{e}\right) \cap\left(\Omega_{R_{4}} \backslash \overline{\Omega_{p}}\right), \pm P_{e}\right)=0 .
$$

Let $R_{4}$ be large enough, then by Lemma 2.2, (3.2) and (3.12), one can obtain

$$
\begin{aligned}
& \operatorname{deg}\left(I-A, \Omega_{R_{4}} \backslash\left[\overline{\Omega_{p}} \cup\left(\left( \pm P_{e}\right) \cap\left(\overline{\Omega_{R_{4}}} \backslash \Omega_{p}\right)\right)\right], \theta\right) \\
& \quad=\operatorname{deg}\left(I-A, \Omega_{R_{4}}, \theta\right)-\operatorname{deg}\left(I-A, \Omega_{p}, \theta\right)-\operatorname{deg}\left(I-A, \pm \stackrel{\circ}{P}_{e} \cap\left(\Omega_{R_{4}} \backslash \overline{\Omega_{p}}\right), \theta\right) \\
& \quad=(-1)^{\gamma}-1-0=-2 \neq 0,
\end{aligned}
$$

which implies that $A$ has at least one fixed point $x^{*} \in \Omega_{R_{4}} \backslash\left[\bar{\Omega}_{p} \cup\left(\left( \pm P_{e}\right) \cap\left(\overline{\Omega_{R_{4}}} \backslash \Omega_{p}\right)\right)\right]$. i.e., BVP (1.1) has at least a sign-changing solution. The proof is completed.

\section{Competing interests}

The author declares that she has no competing interests.

\section{Author's contributions}

The author read and approved the final manuscript.

\section{Acknowledgements}

This work was supported by National Natural Science Foundation of China (No. 11571197), and by Shandong Provincial Natural Science Foundation of China (No. 2016ZRB01076).

\section{Publisher's Note}

Springer Nature remains neutral with regard to jurisdictional claims in published maps and institutional affiliations.

Received: 4 February 2017 Accepted: 5 April 2017 Published online: 20 April 2017

\section{References}

1. Podlubny, I: Fractional Differential Equations. Mathematics in Science and Engineering, vol. 198. Academic Press, New York (1999)

2. Kilbas, AA, Srivastava, HM, Nieto, JJ: Theory and Applicational Differential Equations. Elsevier, Amsterdam (2006)

3. Lakshmikantham, V, Vatsala, AS: General uniqueness and monotone iterative technique for fractional differential equations. Appl. Math. Lett. 21, 828-834 (2008)

4. Jankowski, T: Boundary problems for fractional differential equations. Appl. Math. Lett. 28, 14-19 (2014)

5. Zhang, XG, Liu, LS, Wu, YH, Wiwatanapataphee, B: The spectral analysis for a singular fractional differential equation with a signed measure. Appl. Math. Comput. 257, 252-263 (2015)

6. Zhang, XG, Liu, LS, Wu, YH: The eigenvalue for a class of singular p-Laplacian fractional differential equations involving the Riemann-Stieltjes integral boundary condition. Appl. Math. Comput. 235, 412-422 (2014)

7. Zhang, XQ: Positive solutions for a class of singular fractional differential equation with infinite-point boundary value conditions. Appl. Math. Lett. 39, 22-27 (2015)

8. $\mathrm{Xu}, \mathrm{XJ}$, Jiang, DQ, Yuan, CJ: Multiple positive solutions for the boundary value problem of a nonlinear fractional differential equation. Nonlinear Anal. 71, 4676-4685 (2009)

9. $\mathrm{Xu}, \mathrm{XJ}, \mathrm{Hu}, \mathrm{WW}$ : A new existence results of positive solution for a class of nonlinear fractional differential equation boundary value problems. J. Syst. Sci. Math. Sci. 32(5), 580-590 (2012)

10. Zhang, $X Q$, Wang, $L Q$, Wang, $S$ : Existence of positive solutions for a class of nonlinear fractional differential equations with integral boundary conditions. Appl. Math. Comput. 226, 708-718 (2014)

11. Zhai, CB, Hao, MR: Fixed point theorems for mixed monotone operators with perturbation and applications to fractional differential equation boundary value problems. Nonlinear Anal. TMA 75, 2542-2555 (2012)

12. Liu, YS: Multiple positive solutions of nonlinear singular boundary value problem for fourth-order equations. Appl. Math. Lett. 17, 747-757 (2004)

13. Krasnoselskii, MA, Zabreiko, PP: Geometrical Methods of Nonlinear Analysis. Springer, New York (1984)

14. Guo, DJ, Laksmikantham, V: Nonlinear Problems in Abstract Cones. Academic Press, Boston (1988)

15. Zhang, KM: Non-trivial solutions of fourth-order singular boundary value problems with sign-changing nonlinear terms. Topol. Methods Nonlinear Anal. 40, 53-70 (2012)

16. Deimling, K: Nonlinear Functional Analysis. Springer, Berlin (1985)

17. Zhang, KM, Xie, XJ: Existence of sign-changing solutions for some asymptotically linear three-point boundary value problems. Nonlinear Anal. TMA 70(7), 2796-2805 (2009)

18. Cui, YJ: Uniqueness of solution for boundary value problems for fractional differential equations. Appl. Math. Lett. 51, 48-54 (2016) 